\title{
The microdebrider, a step forward or an expensive gadget?*
}

\author{
Marjolein E. Cornet, Susanne M. Reinartz, Christos Georgalas, Erik van \\ Spronsen, Wytske J. Fokkens
}

Department of Otorhinolaryngology, Academic Medical Centre Amsterdam, Amsterdam, the Netherlands
Rhinology 50: 191-198, 2012

DOI:10.4193/Rhino11.250

*Received for publication:

November 28, 2011

Accepted: February 1, 2012

\section{Summary}

Background: Although the use of the microdebrider (shaver) is well known in endoscopic sinus surgery (FESS), there is lack of evidence from comparative studies focussing on the difference in operating time, intra-operative blood loss and userfriendliness between the microdebrider and traditional operating techniques. In this study we compared the use of the microdebrider to conventional instruments in FESS in these areas.

Methods: A prospective randomised double blind controlled trial in 60 patients with chronic rhinosinusitis with nasal polyposis (CRSwNP) undergoing bilateral FESS. Each subject received FESS using only traditional instruments (Blakseley forceps) on one side and the other side with the additional use of the microdebrider, this way serving as their own control. The primary outcome was operation time, intra-operative blood loss and user friendliness and secondly safety and postoperative healing with a follow-up period at different time points up to three months postoperative.

Results: We found a 37\% longer operating time when operating without a microdebrider. This difference was highly significant. The microdebrider scored significantly higher on every different parameter of user friendliness, except on the preparation of the instrument needed before surgery. For estimated blood loss during surgery we found no differences. Also there was no significant difference in postoperative healing at any point of time.

Conclusions: This study demonstrates that operating patient with CRSwNP with the microdebrider is efficient and that the microdebrider at the same time is safe and easy to use.

Key words: endoscopic sinus surgery, microdebrider, powered instrumentation, nose, paranasal sinuses

\section{Introduction}

Chronic rhinosinusitis (CRSsNP) and chronic rhinosinusitis with nasal polyposis (CRSwNP) are one of the most common health problems these days. The GALEN European health survey reported a prevalence of CRS in Europe around 11\% (1). Besides that CRS also has a major impact on the quality of patient's lives ${ }^{(2)}$. Patients who have CRSwNP frequently need endoscopic surgery, when conservative treatment with topical and oral steroids fails. At the moment approximately 700 sinus surgeries per year per 1 million persons are performed ${ }^{(3)}$.

Although polypectomy has already been described since Hippocrates around 400 BC, nowadays Functional Endosco- pic Sinus Surgery (FESS) is the technique of choice in sinus surgery. The goal of endoscopic surgery is to re-establish normal ventilation and mucus drainage from the sinuses and to resect irreversibly changed mucosa. FESS is considered a safe procedure and meta-analysis of data on complication rates suggest that major complications occur in about $1 \%$ and minor complications in about $5-6 \%$ of the cases ${ }^{(4)}$. Several studies have demonstrated the efficacy of FESS for CRSWNP $(5,6)$. However, like in all fields of surgery surgical techniques have been further refined and with the ongoing evolution in the field of endoscopic mechanisms, new devices are introduced frequently. 
Originally in FESS only the non-cutting Blakesley forceps was used based on the principle of grabbing the polyp and tearing it off. But its use was thought to result in a lot of mucosal trauma and surgical bed scarring. Therefore, one of the first innovations was the cutting forceps, which has been claimed to result in less trauma of the mucosa and therefore better wound healing. In 1992, the thought of further minimizing mucosal trauma led to the introduction of the microdebrider (shaver) by Setliff ${ }^{(7)}$. The microdebrider is a powered rotary shaving device, which originally was used in arthroscopic surgery. It consists of a small rotating blade protected by a blunt end, which can resect tissue that is suctioned into the opening. Because the microdebrider resects tissue very precisely, it minimizes mucosal trauma, which is considered to result in faster healing, compared to traditional instruments. Without the need for removal, the microdebrider supplies continuous suction, enabling the surgeon to maintain a bloodless field while operating. This may improve safety, because the visibility is very important to identify the anatomy is FESS and as well could safe precious operating time. On the other hand with the use of powered instrumentation there are reports published showing a higher incidence of serious complications, like cerebrospinal fluid leak and orbital injury ${ }^{(8,9)}$.

Furthermore, the use of the microdebrider could bring along higher costs, because blades are disposable so they stay very sharp and sometimes more than one blade is needed in one operation ${ }^{(10)}$.

Although the use of the microdebrider is well known, conflicting results are found in literature whether the microdebrider technique is superior, equal or inferior to the traditional techniques. While there are many papers on this subject, there are very few comparative studies and most of them are case reports or retrospective studies instead of randomized controlled trials ${ }^{(11)}$. Besides that most studies include very mixed groups of patients.

The first study that compared traditional techniques with powered instrumentation was conducted in 1996. This was a retrospective, non-blinded study of 250 patients, which reported faster healing with less crusting, and to have less bleeding, synechia formation, lateralization of the middle turbinate, and ostial reoclusion than the traditional group.

There have been only two prospective randomized controlled trials focusing on the healing time after the utilisation of the microdebrider in patients undergoing bilateral FESS ${ }^{(12,13)}$. These two studies found no difference in synechia formation, patency of middle meatal antrostomy and open access tot the ethmoid, and therefore no major advantages compared to standard instruments ${ }^{(12,13)}$. In the study of Sauer et al., the authors even concluded that operating time needed for the microdebrider was significantly longer than with traditional instruments ${ }^{(13)}$. In all studies until now the difference in operating time and therefore cost effectiveness, intra-operative blood loss and post-operative pain, have only been scarcely investigated.

Therefore, we designed this prospective randomized doubleblind (concerning the postoperative outcomes) controlled trial to compare the use of the microdebrider to that of conventional instruments only in FESS. The present paper will first focus on the per-operative parameters and the efficacy of the different operating techniques, evaluating operating time, estimated intra-operative blood loss and user friendliness of the different instruments, and second at the safety and difference in post-operative healing.

\section{Materials and methods}

\section{Patients}

In this mono-centre prospective double-blind randomized controlled trial conducted in the Academic Medical Centre in Amsterdam, the Netherlands, we included 60 patients (120 sides) with bilateral CRSwNP (35 men, 25 women). All participants were over 18 years and the age distribution was between 18 and 72 years old. All patients are diagnosed with strictly symmetrical disease of the paranasal sinuses and had to give written informed consent. Because this study was conducted in a university referral centre, almost all patients had been operated one or several times on the sinuses before. This protocol was approved by the local ethics committee. The inclusion and exclusion criteria are shown in Table 1.

\section{Randomisation}

The subjects who fulfilled the randomisation criteria were randomized to receive FESS using only traditional instruments (straight and curved cutting and blunt Blakesley forceps) on one side and the other side with additional employment of the microdebrider, this way serving as their own control. The side on which the microdebrider was used was randomized using an internet-based randomisation program with a 1:1 ratio.

\section{Study design}

We performed preoperative assessment including clinical and Ear, Nose and Throat (ENT) history, a full ENT examination, CT scans of the sinuses, nasal assessment and evaluation of symptoms. The nasal assessment was done directly preoperative by rigid endoscopy by the surgeon himself before randomisation was revealed. Then the surgery was performed with traditional instruments on both sides and on top of that it was allowed to use the microdebrider on the side indicated by randomisation. Postoperatively, all patients were treated identically including a topical steroid spray and nasal washing 
with saline. A few patients required extra treatment with prednisone or antibiotics. We registered the use of medication during the whole period of follow up.

Follow-up visits where performed at 1, 2, 4 and 6 weeks, and 3 months, postoperatively. During these visits nasal assessment and evaluation of symptoms was done as described later. Furthermore, the adverse events were evaluated during the whole study period ${ }^{(14)}$.

\section{Endoscopic evaluation}

The endoscopic examination included a polyp score for each side separately and scoring of nasal discharge, evidence of oedema, scarring, crust formation for each side in a standardised way per location (nasal cavity, ethmoïd region, region of the infundibulum) according to the Lund-Kennedy scoring system (Table 2) ${ }^{(15)}$.

If preoperative the nose was so obstructed by polyps that the different regions could not be properly scored, we scored 2 points ( $=$ maximum score) for oedema and the rest -2 , meaning this score could not be evaluated. We calculated the mean value of every endoscopic finding separately and compared the means from the microdebrider side with the traditional side. Besides that the total endoscopic score for each instrument at different time points was calculated. We added up all the different mean scores (oedema, nasal discharge, synechiae and crusting) at 4 different time points and compared again the microdebrider with the traditional side.

\section{Evaluation of symptoms}

Evaluation of subjective symptoms was done by validated questionnaires, using pain scores and lateralised symptom scores by means of visual analogue symptom scales (VAS) and a modified Chronic Sinusitis Survey (CSS) form to determine symptoms on both sides (Table 3$)^{(15)}$.

The symptom score using VAS included symptoms of nasal blockage, nasal discharge, sense of smell, headache and purulent discharge for each side with a maximum score of 100 . Furthermore, we calculated a total symptom score by adding up all the separate scores for each side with a maximum score of 500. The modified CSS score for lateralised symptoms included headache, nasal blockage and secretions with a maximum score of 4 for each side separately. The total CSS score was calculated by adding up all the different scores with a maximum of 12 points.

\section{Surgical procedure}

The surgery was performed under general anaesthesia and with traditional instruments on both sides and on top of that it was allowed to use the microdebrider on the side indicated by randomisation. At the side randomised to use traditional instruments only these instruments were used. At the side randomised to use the microdebrider on top of the traditional instruments, the microdebrider was used for the major part of the operation. It was used to remove the polyps, diseased mucosa and part of the bony partitions between the ethmoidal cells. When necessary conventional instruments were used, e.g. to cut thicker bone fragments. All the surgeries were performed by an ENT specialist ( 5 different in total) together with a resident ( 5 different in total). The resident always operated the same amount of time on both sides and the operation was always finished by the ENT specialist himself. The surgeon always started operating on the left side. During the surgery $0^{\circ}$ and $30^{\circ}$ microdebrider blades from Medtronic were used, depending on the anatomical situation. On both sides, we performed standard surgical procedures including uncinectomy, anterior and posterior ethmoidectomy, Draf lla or sphenoidectomy if necessary. If there was any reason for the surgeon during the operation to deviate from the protocol, it was registered in the file. Operating time (in minutes) on the first randomised side was measured when the surgeon started operating and ended

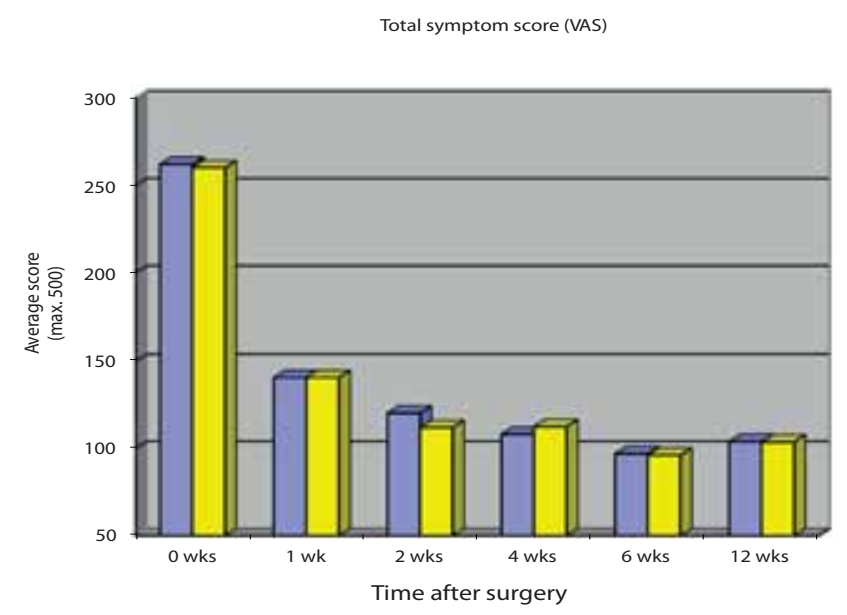

Figure 1. Total symptom score (VAS).

(gray: microdebider; yellow: traditional).

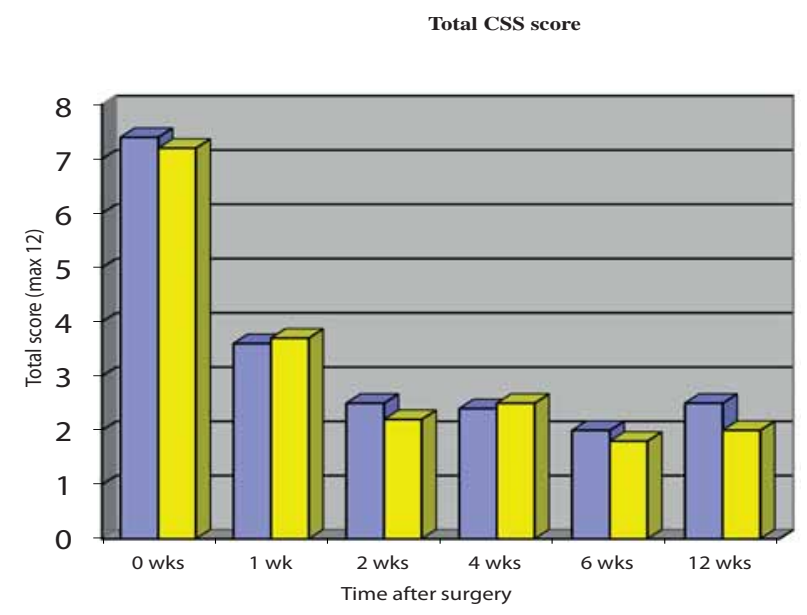

Figure 2. Total CSS score at different points in time after surgery. (gray: microdebider; yellow: traditional). 
when the nasal packaging has achieved haemostatic acceptability on that side.

\section{Blood loss}

Intra-operative blood-loss (in $\mathrm{ml}$ ) was measured on the left (starting side) at the end of the surgery by measuring the fluid in the suction bags. A small merocel packing was put in the left ethmoid to prevent spill over of blood loss when surgery was performed on the right side. All rinsing fluids was accounted for and deducted from the from the total amount of fluids removed by suction during operation. Subsequently the other side was operated with the same measurements at the end.

\section{Questionnaire for the surgeon}

Furthermore, to determine the user-friendliness of the different instruments for the operating surgeon, a questionnaire was completed by the surgeon directly after the operation. This questionnaire contained the following parameters: general use, preparation, reach thoroughness, versatility and handiness. Most of the time, the ENT specialist filled in this form, but if the resident operated the major part of the surgery, he did.

\section{Statistical analysis}

The estimated number of patients needed to show a relevant and significant difference for surgical time comparing the microdebrider side to the conventional side was 60 based on a reduction of $25 \%$ in surgical time. In the analysis, we first tested whether our data were normally distributed. If that was the case, we used the Student's t-test to evaluate statistically significant differences. Significance level was set at $p<0.05$. If the data were not normally distributed, we used the Wilcoxon Signed Rank test.

Over the difference in symptoms score over time after surgery we performed a repeated measurements analysis ANOVA for all different variables.

\section{Results}

\section{Patients}

Patients were male in 58\% ( $n=35)$ and female in $42 \%(n=$ 25). The mean age of all patients was 48 years (range 18 to 72 years). Almost all patients had previous sinus surgery ( $85 \%$ ). Some even had several operations, with a mean of 2.1 operations per patient.

\section{Operative data}

We found a $37 \%$ longer operating time at the side with only traditional instruments (41 [inter quartile range (IQ) range 28 - 49] minutes; $\mathrm{p}<0.001$ ) than on the microdebrider side (30 [IQ range 22-39] minutes) (Table 4). This difference was highly significant.
For the estimated blood loss during surgery, no significant difference was found. It showed a total blood loss (median) in the microdebrider group of 100 [IQ range 43-244] and in the traditional group of 100 too [IQ range $50-180](p=0.94)$ (Table 4).

\section{User friendliness}

The user-friendliness of the two different instruments was analyzed for all the separate questions (Table 5).

These results show that there is a significant difference between all different parameters scored in favour of the microdebrider, except the preparation, which scores significantly higher for the traditional instruments. We observed no differences between the scores of the 5 different residents and 5 different specialists who filled out the forms.

\section{Protocol deviation}

In 11 cases (19\%), the surgeon felt the need to deviate from the protocol by using the microdebrider for a short period on the traditional side. These were all cases where the surgeon felt that he/she was safer using the microdebrider, mostly in cases where polyps were growing in the frontal recess.

\section{Subjective symptoms}

The average total symptom score (VAS) showed an improvement after surgery on the microdebrider side as well as the side with only traditional instruments from a mean score of 260 before surgery in both groups to a final score of 103.9 on the microdebrider side and 103.6 on the traditional side at 3 months. We found no significant differences in total symptom scores after surgery at any point in time (Figure 1). Furthermore, repeated measurements analyses showed no significant differences between microdebrider and traditional side over time.

While analyzing the individual lateralised symptoms scores of nasal blockage, nasal discharge, sense of smell, pain/headache and purulent discharge, we found significantly more purulent discharge at 4 weeks after surgery from the nose on the traditional side (17.4) than on the microdebrider side (14.4) ( $p$ $=0,02$ ). Furthermore, there was an insignificant tendency at 2 weeks postoperative towards more pain on the microdebrider side (16.4 compared with 13.6$)(p=0,09)$. Otherwise no statistically significant differences were found.

We observed an improvement in CSS scores over time, with an mean score difference of 4.9 (preoperatively 7.4 to 2.5 three months postoperatively) points on the microdebrider side and 5.2 (preoperatively 7.2 to 2.0 three months postoperatively) on the side of the nose operated with only traditional instru- 
Inclusion criteria

1) Male or female aged $\geq 18$ years

2) Patients diagnosed with CRSwNP with indication for FESS

3) Written informed consent

4) Symmetrical disease conformed by CT scanning

5) Operation done by ENT-surgeon or experienced resident, as judged by ENT-surgeon supervising resident
Exclusion criteria

1) Patients with any serious or unstable disease

2) Any structural nasal abnormalities (other then polyps), e.g. severe nasal septum deviation

3) Rhinosurgery during past 6 weeks

4) Females who are pregnant

5) Inability to follow instructions within protocol or to attend clinical visits

Table 2. Healing score as evaluated by nasal endoscope.

\begin{tabular}{|llll} 
& \multicolumn{1}{c}{ Characteristics } & Left & \multicolumn{2}{c}{ Right } \\
\hline Nasal Discharge & $(0=$ none, $1=$ clear, 2 = thick, purulent discharge $)$ & $0-2$ & $0-2$ \\
\hline Oedema & $(0=$ absent, $1=$ mild, $2=$ severe $)$ & $0-2$ & $0-2$ \\
\hline Scarring (synechiae) & $(0=$ absent, $1=$ mild, $2=$ severe $)$ & $0-2$ & $0-2$ \\
Crusting & $(0=$ absent, $1=$ mild, $2=$ severe $)$ & $0-2$ & $0-2$ \\
\hline Total endoscopic score & & $0-8$ & $0-8$ \\
\hline Polyps (residual) scored for each side of the nose & $0-3$ & $0-3$
\end{tabular}

$0=$ absent, 1 = within middle meatus, 2 = beyond middle meatus, $3=$ completely obstructing nose .

ments. We found no significant differences between total CSS scores of the different groups after surgery at any of the points in time and also in the repeated measurements over time were no significant differences measured (Figure 2). Besides that, we analyzed all the separate subjective, lateralised CSS scores (headache/pain, nasal blockage and nasal secretions) between the microdebrider - and traditional side and here we also didn't find any statistically significant differences.

\section{Nasal assessment}

We see an improvement of the total endoscopic score over time, both in the microdebrider group and in the traditional group. There is a total endoscopic score at 2 weeks after surgery of 2.4 on the microdebrider side and 2.2 on the traditional side and this score goes down to a score of 1.4 in the microdebrider group and 1.6 in the traditional group at 12 weeks after surgery. We found no significant differences in total endoscopic scores between the microdebrider and traditional instruments at any point in time after surgery and also no differences while analyzing repeated measurements over time.
Furthermore, we analyzed all the detailed endoscopic scores (nasal discharge, oedema, scarring and crusting) for each different side at all time points after surgery. Here we didn't find any significant differences.

\section{Recurrence of polyps /cobblestones}

Endoscopic examination showed no difference in final recurrence rate after 3 months between the microdebrider and the traditional side. We found a rate of $30 \%$ in both groups, however most of these recurred polyps were classified as very small or even cobblestoned mucosa.

Four weeks after surgery we did find a significant $(p=0,04)$ higher recurrence rate of polyps and cobblestones on the traditional operation side (27\%) compared to the microdebrider side $(10 \%)$.

\section{Adverse events}

In 56 patients, a total of 8 minor adverse events occurred. Most of these were small postoperative bleedings only needing an extra Merocell tampon. We found no difference in level of adverse events between the microdebrider and traditional 
Table 3. Symptom score (VAS) and modified CSS score.

\begin{tabular}{|lcc|}
\hline \multicolumn{1}{|c}{ VAS score } & Left & Right \\
\hline Nasal blockage & $0-100$ & $0-100$ \\
\hline Nasal discharge & $0-100$ & $0-100$ \\
\hline Sense of smell & $0-100$ & $0-100$ \\
\hline Headache/pain & $0-100$ & $0-100$ \\
\hline Purulent discharge & $0-100$ & $0-100$ \\
\hline Total symptom score & $0-500$ & $0-500$ \\
\hline \multicolumn{1}{|c|}{ cSS score } & & \\
\hline Headache/pain & Left & Right \\
\hline Nasal blockage & $0-4$ & $0-4$ \\
\hline Secretions & $0-4$ & $0-4$ \\
\hline Total CSS score & $0-4$ & $0-4$ \\
\hline
\end{tabular}

instruments. In one patient, the anterior ethmoidal artery was damaged, resulting in a preseptal hematoma, which resolved by itself without any residual damage. This happened on the side were only traditional instruments were used. There were no cases of any serious adverse events, CSF leak, loss of vision, meningitis or death.

\section{Discussion}

This study shows that using the microdebrider is significantly time saving and more easy to use compared to using only traditional techniques in FESS for patients with CRSwNP. We performed this study to primarily look at the efficacy of the different operating techniques, evaluating operating time, estimated intra-operative blood loss and user friendliness of the different instruments, and secondly at the safety and difference in post-operative healing.

In our study, we found a clear significant difference in operating time, showing that operating with the microdebrider on top of the traditional instruments is faster than without. This can be explained because operating with the microdebrider improves visualization and does not require placing the instrument back into the nose every time the tissue is resected. A recent study from Sauer showed the opposite result: a significant higher average operating time for the microdebrider compared to the traditional side ${ }^{(13)}$. A limitation of the Sauer study was the fact that the microdebrider side was operated only with the microdebrider, not with the microdebrider on top of traditional instruments, which we think is a more realistic approach.

In contrast to other studies, this study showed no difference in blood loss between the microdebrider and traditional instruments. In 1996, Krouse and Christmass described a tendency of decreased bleeding on the microdebrider side ${ }^{(10)}$. However, this was a retrospective study were they analyzed the data of 250 patients who underwent surgery with the microdebrider and 225 patients with traditional instruments.

The fact that we found no decrease in blood loss on the microdebrider side despite the shorter surgical time might be because the microdebrider removes the mucosa in smaller pieces resulting in higher blood loss per $\mathrm{cm}^{3}$ removed tissue. There was a potential spillover of blood loss on the second (right) surgical side. Because the side at which the microdebrider was used was randomized this potential spillover is also random and thus can be neglected in the comparison.

This study focuses on the user friendliness of the different instruments. We found a significant difference in the advantage of the microdebrider between all parameters, except the preparation of the instrument. This means that it is easier to operate with a microdebrider than without in patients with CRSwNP. Only the preparation before using the microdebrider is more work than with the traditional instruments, although this will only take a few minutes.

Earlier claimed theoretical advantages of the microdebrider on postoperative healing time and symptom scores were not confirmed by our study. We did not find any significant differences in post operative healing time at all visits. Nor did we find any significant differences in the subjective total symptom scores. We did see an improvement of total symptom score between one week postoperative and 3 months, but this was the same for both operating techniques. Although the study is rather small, post hoc analysis reveals that also with much larger numbers (> 500 patients per group) significance would not have been reached.

We did observe a significant difference in the recurrence rate of polyps at 4 weeks postoperative. On the microdebrider side were significantly less residual polyps than on the traditional side. At the 3 months follow up visit this difference had disappeared. This finding can be explained because the microdebrider can resect mucosa more precisely, therefore leaving less sick mucosa behind, resulting in less polyps directly postoperative. After a few weeks, this advantage will be decreased because of the additional use of steroids and nasal washing. However, a very likely explanation could be the multiple testing that was done and this result has to be confirmed in other studies.

The literature on potential complications when operating with powered instruments is limited. There is no available literature indicating an increased complication rate with the use 


\begin{tabular}{|c|c|c|c|c|}
\hline & $\begin{array}{l}\text { Microdebrider } \\
\text { median [IQ range] }\end{array}$ & $\begin{array}{c}\text { Traditional } \\
\text { median [IQ range] }\end{array}$ & $\mathbf{Z}$ & $\begin{array}{l}\text { Asymp. Sig. } \\
\text { (2-tailed) }\end{array}$ \\
\hline Operating time (min) & $30[22 ; 39]$ & $41[28 ; 49]$ & $-5,285$ & $<0.001$ \\
\hline Blood loss (ml) & $100[43 ; 244]$ & $100[50 ; 180]$ & $-0,76$ & 0.94 \\
\hline
\end{tabular}

Table 5. User-friendliness of the different instruments.

\begin{tabular}{|lcccc} 
& $\begin{array}{c}\text { Microdebrider } \\
\text { Median [inter quartile range] }\end{array}$ & $\begin{array}{c}\text { Traditional } \\
\text { Median [inter quartile range] }\end{array}$ & $\mathbf{Z}$ & $\begin{array}{c}\text { Asymp. Sig. } \\
\text { (2-tailed) }\end{array}$ \\
\hline General Use & $8[8 ; 9]$ & $7[6 ; 8]$ & $-4,650$ & 0,00 \\
\hline Preparation & $7[7 ; 8]$ & $9[8 ; 9]$ & $-3,000$ & 0,003 \\
\hline Reach & $8[7 ; 9]$ & $7[6.25 ; 8]$ & $-2,631$ & 0,009 \\
\hline Thoroughness & $9[8 ; 10]$ & $8[7 ; 9]$ & $-2,669$ & 0,008 \\
\hline Versatility & $9[8 ; 9]$ & $8[7 ; 8]$ & $-2,953$ & 0,003 \\
\hline Handiness & $9[8 ; 9]$ & $8[7 ; 8]$ & $-5,428$ & 0,000
\end{tabular}

of powered instruments, but in a few case reports potentially dangerous complications are described to occur at a higher rate when operating with the microdebrider ${ }^{(8,16)}$. In this study, we found no difference in complication rate between the microdebrider and traditional instruments. Besides that, there was no difference between the severity in adverse events between both groups. Because in our study not only experienced surgeons but also surgeons in training operated with the microdebrider, we can conclude that the use of the microdebrider is as safe as traditional instruments and can be used by both very experienced as more inexperienced surgeons, as long as in areas of risk the surgeon will operate with more caution.

In 11 cases (19\%) in this study, the experienced surgeon deviated from the protocol by using the microdebrider also on the traditional side. These were all cases where the surgeon felt that he/she was safer using the microdebrider, mostly in cases where polyps were growing in the frontal recess. To make sure that these deviations do not interfere with our results, operating time was recalculated after excluding the cases were deviation took place. Even after excluding these cases operating time was much shorter with the additional use of the microdebrider than without $(p=0,001)$. This supports our overall results.

A strength of this study is that it is prospective and comparing traditional instruments on one side to the microdebrider on top of traditional instruments on the other side, in contrast to most studies performed previously. Besides that, not only very experienced ENT specialists operated, but also ENT surgeons on training. This makes the setting more realistic and fair to compare both techniques.

A limitation of our article is the short period of follow-up, which is only 3 months. Maybe if we analyze the subjects for a longer period of time, there would be differences in final outcome between the microdebrider and traditional instruments. However, the choice for this 3 month period is made because a longer follow up would mainly evaluate the natural evolution of the disease itself, rather than the effects of the two different sets of instruments.

Second, when calculating the operating time at the microdebrider side, we didn't measure the exact time needed to install and remove the microdebrider. To prove that this would make no difference afterwards, we added 3 minutes to the operating time at the microdebrider side and repeated the statistics again to look for significance. Even with these 3 minutes extra time, there was a significance level found of $p=0,001$.

\section{Conclusion}

This study demonstrates that operating patients with CRSwNP with the microdebrider on top of traditional instruments is very time efficient. Besides that the microdebrider is safe and easy to use showed by the significant higher user-friendliness as evaluated by every surgeon after the operation.

Furthermore, there is no difference in intra-operative blood 
loss and post-operative pain or healing time. Our results are encouraging, showing that it could be worthwhile to invest in a microdebrider, saving operating time and meanwhile having good results.

We suggest in the future an analysis of the cost-effectiveness of the use of the microdebrider should be performed, taking into account all the different aspects of the use of the microdebrider such as shorter operating time, extra cost of the disposable blades and general costs of healthcare around the operation.

\section{Acknowledgement}

This study was supported by an unrestricted grant by Medtronic Inc.

\section{Author contributions}

MEC: analyzed the data and wrote the manuscript; SMR, CG, EvS: operated the patients and critically read and commented on the paper. WJF: designed the study, operated the patients, analyzed the data and wrote the manuscript.

\section{References}

1. Hastan D, Fokkens WJ, Bachert C, et al Chronic rhinosinusitis in Europe - an underestimated disease. A GA(2)LEN study. Allergy. 2011; 66: 1216-1223.

2. Bateman ND, Fahy C, Woolford TJ. Nasal polyps: still more questions than answers. Laryngol Otol. 2003; 117: 1-9.

3. Kiwa Prismant. Landelijke Medische Registratie. Kiwa Prismant. 2009. Ref Type: Online Source

4. Hopkins C, Browne JP, Slack R, et al. Complications of surgery for nasal polyposis and chronic rhinosinusitis: the results of a national audit in England and Wales. Laryngoscope. 2006; 116: 1494-1499.

5. Poetker DM, Mendolia-Loffredo S, Smith TL. Outcomes of endoscopic sinus surgery for chronic rhinosinusitis associated with sinonasal polyposis. Am J Rhinol. 2007; 21: 84-88.

6. Toros SZ, Bolukbasi S, Naiboglu B, et al. Comparative outcomes of endoscopic sinus surgery in patients with chronic sinusitis and nasal polyps. Eur Arch Otorhinolaryngol. 2007; 264: 1003-1008.

7. Setliff RC, III, Parsons D. The 'Hummer': New Instrumentation for functional endoscopic sinus surgery. Am J Rhinol. 1994; 8: 275-278.

8. Graham SM, Nerad JA. Orbital complications in endoscopic sinus surgery using powered instrumentation. Laryngoscope. 2003;113: 874-878.

9. Church CA, Chiu AG, Vaughan WC Endoscopic repair of large skull base defects after powered sinus surgery. Otolaryngol Head Neck Surg 2003; 129: 204-209.

10. Krouse JH, Christmas DA, Jr. Powered instrumentation in functional endoscopic sinus surgery. II: A comparative study. Ear Nose Throat J. 1996; 75: 42-44.

11. Hackman TG, Ferguson BJ. Powered instrumentation and tissue effects in the nose and paranasal sinuses. Curr Opin Otolaryngol Head Neck Surg. 2005; 13: 22-26.

12. Selivanova O, Kuehnemund M, Mann WJ Amedee RG. Comparison of conventional instruments and mechanical debriders for surgery of patients with chronic sinusitis. Am J Rhinol. 2003; 17: 197-202.

13. Sauer M, Lemmens $W$, Vauterin T, Jorissen $M$ Comparing the microdebrider and standard instruments in endoscopic sinus surgery: a double-blind randomised study. B-ENT. 2007; 3: 1-7.
14. Rombout J, de VN. Complications in sinus surgery and new classification proposal. Am J Rhinol. 2001; 15: 363-370.

15. Lund VJ, Kennedy DW. Staging for rhinosinusitis. Otolaryngol Head Neck Surg. 1997; 117 (3 Pt 2): S35-S40.

16. May M, Levine HL, Mester SJ, Schaitkin B. Complications of endoscopic sinus surgery: analysis of 2108 patients--incidence and prevention. Laryngoscope. 1994; 104: 10801083.

Marjolein E. Cornet

Department of Otorhinolaryngology

Academic Medical Centre

Amsterdam

1100 DD Amsterdam

The Netherlands

E-mail: m.e.cornet@amc.uva.nl 